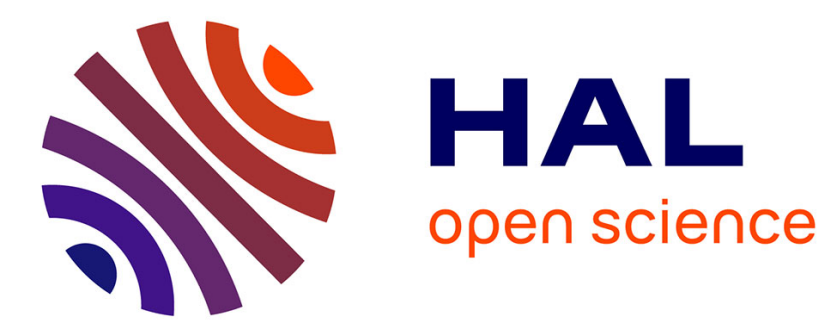

\title{
L'opinion publique en ligne et la mise en ordre du régime chinois
}

Séverine Arsène

\section{To cite this version:}

Séverine Arsène. L'opinion publique en ligne et la mise en ordre du régime chinois. Participations Revue de sciences sociales sur la démocratie et la citoyenneté, 2017, Participer dans le monde chinois: une jeunesse connectée, 17, pp.35-58. 10.3917/parti.017.0035 . hal-01857703

\section{HAL Id: hal-01857703 https://hal.science/hal-01857703}

Submitted on 28 Aug 2018

HAL is a multi-disciplinary open access archive for the deposit and dissemination of scientific research documents, whether they are published or not. The documents may come from teaching and research institutions in France or abroad, or from public or private research centers.
L'archive ouverte pluridisciplinaire HAL, est destinée au dépôt et à la diffusion de documents scientifiques de niveau recherche, publiés ou non, émanant des établissements d'enseignement et de recherche français ou étrangers, des laboratoires publics ou privés. 


\title{
L'opinion publique en ligne et la mise en ordre du régime chinois
}

\author{
Séverine Arsène
}

\section{Résumé}

Cet article retrace les doctrines formulées par les autorités sur l'opinion publique en ligne de la fin des années 1990 à aujourd'hui. À travers elles, se dessine le pari, pris par l'administration $\mathrm{Hu}$, que cette opinion publique, canalisée, puisse être mise au service d'un mode de gouvernement technocratique, et instrumentalisée dans la mise au pas d'un appareil politico-administratif difficile à maîtriser. Dans une période plus récente, le traitement de l'opinion publique s'inscrit dans un contexte de concentration des pouvoirs autour de la personne de Xi Jinping, où la censure est revendiquée par le pouvoir, avec un retour à une conception plus conservatrice du rapport aux médias.

$$
* * *
$$

\section{Introduction et contexte}

Dès les années 1990, le gouvernement chinois a fait le choix de développer massivement les infrastructures et les services sur Internet, faisant de la Chine le premier pays en nombre d'internautes, avec plus de 731 millions d'internautes en janvier 2017, soit $53 \%$ de la population (CNNIC, 2017). Cette stratégie s'est dessinée alors même que la volonté de maintenir le statu quo politique était fermement réaffirmée. Internet était ainsi présenté comme un levier de développement économique, octroyé à la population en échange de sa docilité politique.

À l'heure où le développement des échanges en ligne était majoritairement considéré comme une opportunité d'expression contestataire, ce pari semblait particulièrement risqué. Bien sûr les dissidents chinois se sont très tôt saisis d'Internet pour se mettre en réseau et partager des idées et des ressources. De nombreux groupes de défense des droits ont ouvert des sites où ils fournissent une aide précieuse aux citoyens victimes d'injustices. Plus généralement, les citoyens ordinaires y ont trouvé un espace où exprimer leurs préoccupations et leurs indignations (Yang, 2009). Ils ont ainsi contribué à former une « opinion publique en ligne » qui exerce un poids croissant sur les autorités chinoises (Lagerkvist, 2005a ; Xiao, 2008), même s'il s'agit d'une parole largement contrainte et «cadrée » par la censure et par les normes sociales propres aux classes moyennes urbaines (Arsène, 2011b).

Pourtant, l'effort de développement d'Internet par les autorités chinoises ne s'est pas démenti au cours des deux dernières décennies. Ce choix peut en partie s'expliquer par la mise en place d'infrastructures de censure et de contrôle destinées à canaliser les mouvements contestataires en ligne (ONI, 2012). Les usages les plus ouvertement critiques du gouvernement chinois sont systématiquement censurés, et la Chine compte de nombreux cyberdissidents en prison. De nombreux sites Internet hébergés à l'étranger sont bloqués, et les sites domestiques sont soumis à une réglementation 
très restrictive qui les oblige à censurer les publications de leurs utilisateurs. La censure s'accompagne d'une propagande de plus en plus sophistiquée et de tactiques d'influence des échanges en ligne (Bandurski, 2008a ; A. K. Li, 2015).

Cependant, l'attitude du gouvernement chinois vis-à-vis de l'opinion publique en ligne ne se résume pas à une course-poursuite entre les censeurs et les citoyens contestataires. J'entends montrer dans cet article qu'elle s'inscrit également dans une stratégie plus générale d'encouragement, de canalisation et, souvent, d'instrumentalisation de la participation politique des citoyens.

La notion de participation doit ici s'entendre dans un sens très large, compte tenu du faible nombre de canaux par lesquels les citoyens sont en mesure de s'exprimer et des moyens détournés qu'ils emploient pour y parvenir, ainsi que des mesures prises par les autorités pour faire taire, canaliser, détourner, influencer ou instrumentaliser cette parole politique. Dans le contexte chinois, on ne peut limiter la participation politique aux seules «activités des citoyens destinées à influencer plus ou moins directement la sélection du personnel politique ou les décisions qu'ils prennent », ni même à une définition plus large incluant des «activités se déroulant en dehors (ou à l'encontre) des institutions politico-administratives » (Monnoyer-Smith, Wojcik, 2014, p. 7). En effet, les manifestations, grèves, pétitions sont généralement interdites ou sévèrement réprimées en Chine, et relèvent donc déjà de formes d'engagement très avancées. La participation politique dans un tel contexte peut donc prendre des formes extrêmement variées et fluides, depuis la publication d'opinions plus ou moins expertes, de témoignages, de réactions émotionnelles, d'enquêtes (pensons aux citoyens-journalistes). Elle comprend également des pratiques et conversations quotidiennes, où la critique peut se dissimuler et dont elle peut aussi émerger spontanément, à travers diverses formes d'expression, dont l'ironie, l'humour, l'indignation par exemple, divers éléments qui relèvent d'une «politique des styles de vie » (lifestyle politics, Bennett, 1998).

Réalisant l'importance vitale de ces dimensions pour assurer la stabilité sociale et la pérennité du régime, les autorités chinoises ont mis en place au cours des années 2000 de nombreux dispositifs destinés à mesurer l'opinion publique (People's Daily Online, 2013), à détecter les sujets de préoccupation de la population, ou encore à recueillir des opinions sur des projets d'infrastructures publiques (Leib, He, 2006), que cela soit en ligne ou hors ligne. Les initiatives de e-gouvernement sont particulièrement encouragées à l'échelle nationale, et de nombreuses initiatives sont déployées dans les différentes localités à des fins de modernisation de l'action publique (Schlæger, 2013). Certains chercheurs ont appelé «contrôle 2.0 » ou «autoritarisme en réseau » l'incorporation de l'opinion publique en ligne dans l'action des autorités, comme moyen de surveillance et comme source d'information très précise sur l'état de l'opinion (des classes connectées), devenant ainsi un instrument d'anticipation et d'amélioration de l'action publique, et venant in fine soutenir la légitimité du Parti (MacKinnon, 2011 ; Tsai, 2016).

Ainsi, le traitement de l'opinion publique en ligne s'insère dans la problématique de la « résilience »du régime autoritaire (Nathan, 2003), et dans la réflexion autour de la compatibilité entre un régime autoritaire et l'expression de la société civile sous diverses formes. Certains auteurs proposent, par exemple, la notion «d'autoritarisme consultatif » (Teets, 2013) ou «délibératif» (He, Thøgersen, 2010) pour décrire l'évolution des relations entre autorités locales et $\mathrm{ONG}$, ou la recrudescence d'initiatives de consultation des citoyens. Des travaux récents montrent ainsi le caractère «hybride» des régimes autoritaires comme la Chine, en ce que diverses formes de participation, jusque-là considérées comme l'apanage du fonctionnement 
démocratique, font partie intégrante de leurs modes de gouvernement. Par exemple, Froissart (2014) montre dans un dossier spécial du Journal of Civil Society que divers «mécanismes de dépolitisation permettent à des formes de conflit organisé de coexister avec l'autoritarisme, voire de le renforcer ». Dans cet article, je m'attacherai donc à retracer, de manière diachronique, la conception du pouvoir et de la relation entre l'État et la population qui se dessine au travers des doctrines formulées par les autorités sur l'opinion publique en ligne et la manière dont elle doit être traitée, depuis les années 1990 au cours desquelles Internet a commencé à se développer en Chine. Je prêterai une attention particulière à la manière dont Internet s'inscrit dans des doctrines et des pratiques préexistantes au sein du Parti communiste chinois sur le rôle des médias dans la gestion de la question de l'opinion publique, dont il souligne les limites et pousse à la transformation.

À cette fin, je m'appuie notamment sur le travail de veille et de documentation mené de longue date par David Bandurski et l'équipe du China Media Project à l'université de Hong Kong. Leur attention spécifique aux mots-clés qui jalonnent les discours officiels concernant le secteur des médias et de la culture, tels que "guidage de l'opinion publique » et «supervision par l'opinion publique », offre un éclairage très important sur cette question. Je recours d'autre part, de manière synthétique, aux travaux sur l'économie politique des médias en Chine (Xiaoling Zhang, 2011; Zhao, 2008). Je m'appuie également sur les textes réglementaires qui encadrent le développement d'Internet jusqu'à une période récente, ainsi que sur des déclarations officielles et des éditoriaux publiés dans la presse officielle chinoise (Quotidien $d u$ Peuple, Global Times par exemple).

\section{Entre « direction de l'opinion publique » et « supervision par l'opinion publique » (1989-2006)}

Dans les premières années, le développement d'Internet a été essentiellement perçu comme une extension des possibilités de publication d'informations auprès du grand public. Ainsi la mise en place des infrastructures d'Internet et la régulation de leurs usages ont-elles été initialement le reflet de la doctrine conçue à la fin des années 1980 autour des médias dits traditionnels que sont la presse écrite, la radio et la télévision, qui réside principalement dans les notions complémentaires de «guidage » de l'opinion publique et de «supervision par» l'opinion publique. Dans ce paysage, Internet est cependant progressivement devenu un élément essentiel, en permettant de publier, mais aussi de partager et de commenter des informations. Il a également eu un impact très important sur les pratiques journalistiques dans les autres médias, avec une circulation croissante des contenus entre les blogs, les forums et la presse, laquelle est également très présente en ligne. Cela conduit les autorités à raffiner sans cesse leurs techniques de contrôle et de propagande en ligne.

"Guidage de l'opinion publique » (yulun daoxiang) et « supervision par l'opinion publique » (yulun jiandu) dans un secteur des médias livré au marché

Le terme de «guidage de l'opinion publique» est élaboré par le gouvernement chinois en 1987 et il est mobilisé abondamment à la suite du massacre de Tiananmen, dans un mouvement de reprise en main idéologique du pays qui passe par un contrôle accru des médias. Il s'agit non seulement de rappeler aux médias leur rôle de courroie 
de transmission de la propagande officielle et de réaffirmer l'interdiction formelle de s'écarter de la ligne politique définie par le Parti, mais aussi de trouver des moyens d'intéresser les lecteurs par des contenus plus susceptibles de retenir leur attention. Le recours au terme «opinion publique » signifie ici que les médias doivent jouer un rôle dans l'influence de l'opinion publique, c'est-à-dire non pas la refléter, mais trouver des moyens de fixer pour eux l'agenda politique (Chan, 2007, p. 548).

Ce terme devient particulièrement central en 1992 dans une résolution du Comité central du PCC concernant le renforcement et l'amélioration de la propagande et du travail idéologique (Bureau politique du Comité central du Parti communiste chinois, 1992), puis en 1994 dans un discours à la conférence nationale sur la propagande et le travail idéologique.

En 1996, lors d'une visite au siège du Quotidien du Peuple, Jiang Zemin introduit une série de réformes du secteur des médias. Ces réformes importantes consistent essentiellement à introduire des éléments d'économie de marché dans ce secteur jusque-là entièrement contrôlé par le Parti et dédié à relayer le discours officiel, afin d'en faire un secteur plus dynamique, moderne et surtout rentable. Si la politique éditoriale des titres de presse reste sous la responsabilité de leurs organismes de rattachement au sein de l'appareil politico-administratif, il est désormais possible de développer des rubriques et autres suppléments plus attractifs pour les lecteurs, de manière à attirer des annonceurs (Zhao, 2008). Cette réforme est au fond une application au secteur des médias de la ligne générale qui consiste à encourager l'entreprise privée et le développement économique, sans toucher au statu quo politique. Dans le secteur des médias, cet équilibre est cependant fragile. Il s'agit de maintenir la ligne du Parti, tout en trouvant les angles et le langage propres à toucher et influencer véritablement une opinion rompue au décryptage de la propagande officielle. L'enjeu est de traiter, dans la mesure du possible, des véritables sujets de préoccupation des lecteurs et des auditeurs, de façon à rendre plus efficace encore le travail idéologique en faveur du Parti.

Compte tenu de ce positionnement, les journalistes de la presse écrite et audiovisuelle se trouvent en position privilégiée pour effectuer une veille serrée des attentes et des préoccupations de la population, dans un contexte d'instabilité sociale et d'inégalités croissantes. Pour souligner ce rôle (déjà ancien) de vigie de l'opinion publique au service du Parti et de la définition de la ligne politique, Jiang ravive la notion de «supervision par l'opinion publique ». Il déclare par exemple en 1994 que «la supervision par l'opinion publique doit être au service des efforts du Parti et du gouvernement dans la résolution des vrais problèmes, la promotion de l'unité du peuple et la sauvegarde de la stabilité sociale ${ }^{1}$ (Bandurski, 2007). Les médias sont ainsi chargés de recueillir les doléances de la population à l'égard des dirigeants, et de les faire remonter en vue d'une amélioration des politiques publiques et de la propagande, toujours dans le souci du maintien de la stabilité politique. Ils sont ainsi complémentaires d'une série d'autres dispositifs destinés à faciliter les relations entre la population et les autorités et la résolution des conflits, tels que les Bureaux des lettres et des visites (Thireau, Hua, 2010), ou des dispositifs de médiation mis en place auprès des tribunaux (Hu, Zheng, 2016), qui ont également vocation à maintenir la stabilité. Il n'est pas question, cependant, de soumettre véritablement l'action des autorités à un quelconque tribunal de l'opinion ni de mettre en place une véritable transparence. Les informations ainsi recueillies sont exclusivement à l'usage du Parti et n'ont pas vocation à être publiées.

\footnotetext{
${ }^{1}$ Je traduis à partir de l'anglais.
} 
Ce positionnement vis-à-vis des médias est maintenu et rappelé régulièrement tout au long du mandat de Jiang et lors du premier mandat présidentiel de $\mathrm{Hu}$ Jintao (20022012). On peut par exemple lire en 2003 sous la plume de Li Changchun, secrétaire du Département de la propagande du Parti communiste chinois, que le travail de propagande aurait pour objectif principal d'unifier «l'esprit» du Parti avec celui de l'opinion publique, ce qui doit se traduire par trois formes de «proximité »: «avec la réalité, avec les masses, avec la vraie vie » (C. Li, 2003).

Cela se traduit par exemple par l'ouverture en 2004 d'un Bureau de l'opinion publique au sein du Département central de la propagande, ce qui permet alors de coordonner plus efficacement les efforts de mesure de l'opinion publique de ce département (Brady, Wang, 2009, p. 785).

\section{Le « guidage de l'opinion publique » en ligne}

La mise en place des infrastructures et de la réglementation d'Internet au cours des années 1990 reflète la ligne générale adoptée pour l'ensemble des médias. En Chine, les premières plateformes d'échange sur Internet sont mises en place dans le courant des années 1990. En 1997, on compte 620000 internautes qui peuvent visiter 1500 sites chinois environ, ce qui reste encore très limité. Les éléments interactifs de cet Internet balbutiant consistent essentiellement en des listes de diffusion par mail, puis des messageries instantanées et des forums de discussion (bulletin board systems), souvent internes à des universités ou des organismes de recherche. Ils ne seront ouverts au grand public, ou hébergés sur de grands portails généralistes, qu'au tournant des années 2000. Les blogs se développent au début des années 2000, puis les réseaux sociaux de manière plus générale, avec notamment la plateforme QQ qui réunit différentes fonctions de page personnelle, de réseau social, de partage d'informations et de messagerie personnalisée. À partir de 2010, aux blogs succéderont les plateformes de microblogs, nommées weibo en chinois, et qui accentuent encore la rapidité de la circulation de l'information en ligne.

Cependant, ce développement d'Internet est strictement encadré pour garantir que les informations publiées en ligne restent sous contrôle. Dès que la décision est prise de laisser des entreprises proposer au grand public une offre commerciale d'accès à Internet, entre 1994 (Conseil des affaires de l'État, 1994) et 1996 (Conseil des affaires de l'État, 1996), la législation est adaptée en conséquence (voir aussi Tan, Mueller, Foster, 1997). Sur le plan des infrastructures, il est interdit de construire un accès au réseau indépendamment des quelques grandes entreprises agrémentées pour le faire. Le nombre d'interconnexions avec l'international est limité, ce qui permet de créer des points de passage obligés au niveau desquels des systèmes de filtrage et de blocage des données sont installés. Les fournisseurs d'accès à Internet sont des entreprises à capitaux exclusivement chinois, majoritairement sous contrôle de l'État, ce qui permet des interventions régulières aussi bien sur le plan industriel (fusions, divisions, restructurations majeures en vingt ans) que sur le plan politique. Les utilisateurs, de leur côté, doivent se déclarer auprès du bureau de police local pour pouvoir disposer d'une connexion. Ces mesures sont diversement appliquées localement, mais elles sont clairement destinées à organiser un Internet aussi centralisé que possible, afin de permettre l'intervention de la censure.

À mesure que les services les plus interactifs se développent et permettent la recrudescence de l'expression des individus en ligne, les mesures de contrôle sont progressivement adaptées et étendues. En 2000, une nouvelle série de régulations est publiée (Conseil des affaires de l'État, 2000a, 2000b). Entre autres mesures, la 
fourniture de services en ligne est soumise à une licence délivrée par le ministère de l'Industrie de l'information (MII), seuls les organes de presse officiels peuvent publier des informations en ligne, les investissements étrangers sont strictement limités, etc. Ces règles seront constamment complétées et amendées dans les années suivantes à mesure que de nouveaux services se développent (régulation des cybercafés, enregistrement de l'identité des internautes, etc.).

La régulation de la fourniture d'informations est un point particulièrement central dans ces mesures, qui signale clairement l'alignement d'Internet sur les autres organes de presse et soumet ces sites à la censure effectuée par l'Administration générale de la presse et des publications (GAPP).

Certes, les échanges informels entre les internautes sur les forums ou les messageries instantanées ne relèvent pas de la catégorie des services d'information. Cependant, il n'est pas question de laisser se développer une parole publique incontrôlée. Dans ce domaine, les systèmes de licence et d'autorisation dessinent le principe de responsabilisation des intermédiaires, qui consiste à faire peser la responsabilité des activités en ligne sur les opérateurs, de sorte qu'ils exercent eux-mêmes la censure, par exemple en embauchant des modérateurs qui effacent les contributions des internautes en cas de non-respect des règles de bienséance mais aussi en cas de propos politiquement sensibles. Ils peuvent recevoir à cet effet des consignes informelles émanant plus ou moins directement du Département de la propagande du Parti ou du ministère de la Sécurité publique. Ces mesures ont une efficacité variable, dans la mesure où elles s'avèrent rapidement contournables, mais elles produisent également un effet d'intimidation qui peut conduire certains modérateurs à sur-censurer les contenus et les internautes à s'autocensurer (Arsène, 2011a).

Ainsi Internet se développe-t-il, du point de vue des autorités chinoises, comme un espace où l'information est portée au plus près de la population, dans des formats plus interactifs, qui leur permettent de la relayer, de se l'approprier de diverses manières, voire de la compléter ou de la commenter et de l'enrichir, mais à la condition expresse de ne pas transgresser le principe central selon lequel l'opinion doit rester cantonnée au sein d'un domaine délimité par la propagande officielle.

\section{Vers la « canalisation de l'opinion publique »: Internet comme instrument de gestion technocratique (2007-2012)}

\section{Contexte}

La première moitié de la décennie 2000 montre pourtant les limites des mesures prises en vue de garantir le contrôle de l'opinion publique en ligne, à mesure que les internautes s'approprient les forums de discussion puis, vers 2005, les blogs. Dès le tournant du siècle, des mobilisations à caractère nationaliste échappent au contrôle des autorités (Wu, 2007). En 2003, le scandale Sun Zhigang (Thireau, Hua, 2005) montre qu'Internet peut représenter une chambre d'écho telle qu'à partir d'un simple fait divers concernant la mort d'un migrant, une mobilisation portée notamment par des journalistes et des juristes aboutit à la modification de la loi. Devant ces premières réussites, de plus en plus d'internautes s'enhardissent et on assiste à une recrudescence de scandales en ligne, que les outils de censure ne peuvent étouffer complètement. En effet, en dépit de la sophistication croissante de l'arsenal réglementaire et technique dont disposent les censeurs, les stratégies des internautes pour contourner la censure, l'imprévisibilité des affaires et la viralité des messages en 
ligne rendent évident que ce jeu du chat et de la souris ne peut pas aboutir à un contrôle parfaitement efficace de l'opinion publique en ligne.

Or Hu Jintao aborde son deuxième mandat, à partir de 2007, dans un contexte politique particulièrement tendu. La Chine prépare les Jeux Olympiques de Pékin (2008) et l'Exposition universelle de Shanghai (2010), qui sont conçus comme le symbole de l'avènement de la Chine en tant que grande puissance mondiale. L'enjeu en termes de communication et de propagande est central, alors que les tensions sociales qui traversent le pays sont plus exacerbées que jamais. Les «mouvements de masse » se multiplient tant que les statistiques officielles ont cessé d'être publiées en 2006 (on estime le nombre d'émeutes locales à 180000 en 2012 : Bloomberg, 2011). En 2008 de violentes émeutes éclatent au Tibet, puis en 2009 au Xinjiang. Le contexte international est également préoccupant pour les autorités chinoises avec le déclenchement du Printemps arabe en 2011, qui leur fait craindre une « révolution de jasmin $\gg$ chinoise.

Dans ce contexte, la question de l'opinion publique fait l'objet de débats enragés au sein du Parti. Pour certains, elle ne va pas assez loin, alors que pour d'autres elle représente un risque majeur de déstabilisation du régime. Un groupe de vingt-trois anciens dirigeants de haut rang rend ainsi publique en 2010 une lettre ouverte au comité permanent du Congrès national du peuple, demandant la fin des limitations de la liberté d'expression en Chine (et notamment de certaines mesures d'influence de l'opinion publique en ligne) (Bandurski, 2010c). Au contraire, d'autres voix se font entendre au sein du Parti pour renforcer la censure. En réponse à la lettre ouverte, un éditorial du journal À la Recherche de la Vérité est ainsi intitulé en 2010 «Perdre l'opinion publique, c'est tout perdre » et s'inquiète d'une possible chute du régime (Bandurski, 2010b).

La réponse de l'équipe dirigeante consiste à proposer une forme de modernisation du contrôle de l'opinion publique, qui prenne acte des transformations irrémédiablement introduites par le développement d'Internet, tout en assurant que le Parti puisse conserver une position dominante dans ce nouveau paysage.

\section{La reconnaissance symbolique de l'opinion publique en ligne}

L'année 2008, particulièrement tendue politiquement, est justement le moment que choisit $\mathrm{Hu}$ Jintao pour reprendre en main la question des médias. À l'occasion du soixantième anniversaire du Quotidien $d u$ Peuple, il y donne, à son tour, une importante visite (Bandurski, 2008b). Cette visite entérine la reconnaissance de l'existence d'une forme d'opinion publique qui s'exprime sur le web. Elle est d'abord médiatisée à travers l'exercice de chat avec des internautes auquel se prête $\mathrm{Hu}$ : il est photographié souriant, devant un écran d'ordinateur, répondant aux questions anodines de trois internautes que l'on devine soigneusement sélectionnés (People's Daily Online, 2008). Cette photographie, publiée par le Quotidien du Peuple, a été reprise abondamment dans la propagande officielle. Je l'ai notamment retrouvée en 2009 sur le stand du Quotidien du Peuple à Paris, lors de la Fête de l'Humanité, illustrant le texte suivant : «La participation et la délibération du peuple dans les affaires publiques via Internet a connu des progrès. Cela est encouragé par le président $\mathrm{Hu}$ Jintao, le premier ministre Wen Jiabao ainsi que les dirigeants de différents ministères et provinces. Le respect à l'égard des avis des internautes traduit le développement de la démocratie de la société chinoise ».

Les dirigeants prennent ainsi acte de l'impossibilité d'étouffer totalement les affaires et de l'attachement croissant de la population chinoise à la possibilité de s'exprimer 
en ligne. Dès 2006 par exemple, Wang Guoqing, vice-ministre du Bureau de l'information au Conseil des affaires de l'État (le gouvernement chinois), déclarait qu'il était naïf de vouloir étouffer des informations (Bandurski, 2006). En 2012, cette position est réitérée par un éditorialiste du très officiel Global Times dans un article intitulé «Punir la critique est obsolète dans la Chine d'aujourd'hui » (Yu, 2012). De même, Xuyang Jingjing, journaliste au Global Times, est autorisé à commenter de manière relativement critique le renforcement du contrôle de l'Internet à Pékin, dans un article repris dans la version en ligne du Quotidien du Peuple. Il souligne que les offenses susceptibles d'être punies sont mal définies (ce qui est inconstitutionnel) et appelle les autorités à faire preuve de plus de transparence afin d'éviter en amont l'émergence de telles offenses. Il cite également Yu Guoming, directeur de l'Institut de l'opinion publique à l'université du Peuple, qui déclare que «les gens ont le droit de superviser le gouvernement et sont en droit d'exprimer leurs émotions, sentiments ou opinions, quelles que soient ces opinions » (Xuyang, 2012).

\section{Vers un contrôle plus «moderne » : la «canalisation de l'opinion publique»}

Il ne s'agit cependant pas d'ouvrir la voie à une expression véritablement libre de l'opinion publique. Si le discours de Hu Jintao au Quotidien du Peuple reconnât l'importance de l'opinion publique, il réaffirme néanmoins la nécessité de la contrôler en modernisant les méthodes de «guidage» afin de les rendre à la fois plus supportables pour les internautes et plus efficaces dans la perspective de garantir la stabilité politique.

Cette modernisation est marquée par une inflexion du langage utilisé pour désigner la stratégie de contrôle de l'opinion publique. Hu Jintao introduit ainsi, en sus de la notion de «guidage », celle de «canalisation de l'opinion publique » (yulun yindao), qui deviendra dominante à partir de la crise qui secoue le Tibet en 2008 (Bandurski, 2010a).

Cette évolution se traduit par des mesures de contrôle plus sophistiquées qu'auparavant. Certaines mesures perpétuent la logique précédemment instaurée. L'adaptation de la réglementation à l'évolution des services se poursuit, avec notamment la généralisation du «système de noms réels » qui oblige les plateformes de microblogs à enregistrer le numéro de carte d'identité ou de téléphone mobile de leurs utilisateurs. Les différents systèmes de filtrage sont en constante évolution, permettant de bloquer ou ralentir des sites de manière plus subtile et surtout avec une réactivité plus grande en fonction de l'actualité.

Cependant, l'évolution du contrôle de l'opinion publique en ligne se manifeste surtout par une augmentation significative de la propagande en ligne, en quantité comme en qualité. La consigne est désormais donnée de mettre en place des dispositifs qui permettent de repérer au plus tôt les crises, afin d'intervenir avant qu'elles ne prennent une ampleur trop importante et d'y répondre par des stratégies de communication élaborées. Chaque administration, chaque cellule du Parti à travers le pays sont encouragées à mettre en place des équipes chargées d'élaborer cette communication, qui peut prendre des formes très officielles et transparentes, comme l'ouverture de sites de e-gouvernement ou la tenue de fils d'information sur Weibo, mais aussi des formes plus souterraines, comme le fait de payer des équipes d'anonymes pour noyer les conversations en ligne de commentaires destinés à influencer l'opinion en faveur des autorités, une technique de communication 
nommée «astroturfing», et que David Bandurski compare à une «guérilla » (Bandurski, 2008a).

Dans cette perspective, Internet est tout d'abord considéré comme un instrument de mesure exceptionnel de l'état de la société et des préoccupations des citoyens. Les travaux scientifiques chinois explorant les différents moyens de mesurer l'opinion publique en ligne se multiplient. De même, l'idée d'utiliser Internet comme support de dispositifs de consultation de la population se développe, avec la multiplication des sondages en ligne. L'objectif de mener une veille serrée de l'opinion, destinée à produire une analyse fine de l'état de la société dans un objectif d'amélioration des politiques publiques, se fait jour à côté, sinon en substitution, de l'objectif de surveillance de la contestation politique. La création en 2008 de l'Observatoire de l'opinion publique au sein du Quotidien $d u$ Peuple marque une forme d'aboutissement de cette logique. L'observatoire mobilise les techniques les plus modernes d'analyse des contenus en ligne, afin de procéder à une expertise des tendances qui traversent la société chinoise (A. K. Li, 2015, p. 17).

Ainsi l'expression de l'opinion publique en ligne, dûment canalisée, peut-elle être conçue comme un instrument au service des autorités en vue de l'amélioration de leurs pratiques de gouvernance, et pas nécessairement ou pas seulement comme un risque de contestation politique. Les entretiens avec des internautes ordinaires que j'ai menés à Pékin en 2006 et 2007 (Arsène, 2011b) m'avaient permis de constater que cette conception était partagée également par bon nombre d'internautes. Un internaute comparait ainsi Internet à une entreprise, dans laquelle l'expression est un instrument de gestion au service des dirigeants :

«En fait Internet c'est comme une entreprise. Les internautes sont comme les employés, et les dirigeants ont intérêt à entendre l'avis des employés, il n'y a que comme ça que l'entreprise peut se développer, n'est-ce pas ?»

Un autre explique très clairement qu'Internet est un moyen de connaître l'opinion de la population et d'adapter ses mesures en conséquence :

« Ça a aussi de l'influence sur le gouvernement, il doit plus tenir compte de l'opinion des masses et de ce que pensent les gens ordinaires. Avant ils avaient très peu de contacts avec les gens ordinaires, ils ne pouvaient les contacter que dans le cas des "lettres et visites". Avec Internet ils peuvent entendre la voix des gens de toutes les couches, ils peuvent atteindre directement des choses vraies, ils peuvent directement les voir. [...] Ça a raccourci le temps de résolution des problèmes entre les gens ordinaires et le gouvernement. »

La vision portée ici est donc celle d'un appareil dirigeant qui n'abandonne pas ses prérogatives au peuple, mais qui s'appuie sur les avis émis par celui-ci pour améliorer son action et sa crédibilité, garantissant ainsi la légitimité de son maintien au pouvoir. La parole ordinaire des internautes deviendrait ainsi une forme de «participation » involontaire et inconsciente au service de la gouvernance du Parti communiste chinois.

\section{Légitimation du pouvoir et mise en discipline des autorités locales}

Cette évolution du traitement de l'opinion publique en ligne est nommée « contrôle 2.0 » par les chercheurs du China Media Project de Hong Kong. Rebecca MacKinnon parle, elle, d' "autoritarisme en réseau» (MacKinnon, 2011). Si cette terminologie rend compte du rôle sans doute crucial joué par la circulation de 
l'information sur Internet dans ce processus, elle perpétue cependant une image quelque peu monolithique de l'État et restitue mal la manière dont il affecte le fonctionnement du régime chinois.

D'une part, ainsi que je l'ai mentionné plus haut, l'opportunité d'offrir une marge de manœuvre plus grande pour l'expression des opinions en ligne ne fait pas l'unanimité au sein de l'appareil du Parti, et d'autre part les inquiétudes sont grandes au sujet d'une possible déstabilisation du régime dans le contexte de tensions sociales fortes de ces dernières années. Les éditoriaux des grands organes de presse se font régulièrement l'écho des visions plus ou moins conservatrices sur ce point.

Ces réticences, présentes à tous les échelons de l'appareil politico-administratif, se conjuguent avec une relative appréhension vis-à-vis des outils eux-mêmes et à une très faible compétence des personnels chargés de mettre en place les politiques sur le terrain, dans les municipalités, les bureaux de police ou les administrations locales, même si les efforts de formation des personnels tendent à faire évoluer cette situation. Sur le terrain, on observe de fait une grande diversité dans les stratégies de gestion de l'opinion publique en ligne mises en œuvre à l'échelle locale, avec parfois la survivance presque incompréhensible de pratiques qui sont désormais considérées d'un autre âge.

Par exemple, lors d'un dramatique accident affectant deux trains à grande vitesse à proximité de Wenzhou en 2011, la première réaction des autorités locales fut d'enterrer les wagons, avant même que les corps des victimes puissent être évacués et une enquête diligentée, et d'interdire aux médias de couvrir l'affaire. Le scandale provoqué par cette attitude sur les réseaux sociaux a cependant permis d'interrompre cette destruction des éléments de preuve et d'obtenir des excuses officielles ainsi que la visite du Premier ministre Wen Jiabao (Murphy, 2011).

La mise en place concrète de la stratégie de canalisation de l'opinion publique en ligne se fait donc à tâtons, au fil des scandales et des affaires. Elle est tributaire de la vision plus ou moins conservatrice portée par les responsables de la propagande à l'échelle centrale comme à l'échelle locale. Elle fait également l'objet de conflits récurrents entre les différents échelons de l'appareil politico-administratif. En effet, si les autorités centrales tendent à promouvoir des pratiques de transparence accrues destinées à promouvoir la stabilité politique à long terme, les autorités locales font directement les frais des dénonciations de scandales et sont plus facilement tentées de recourir à des méthodes de censure considérées comme plus « archaïques ».

L'un des cas les plus exemplaires de la manière dont la gestion de l'opinion publique peut opposer le niveau local et le niveau central est celui des émeutes de Weng'an (D. Li, 2008), qui ont eu lieu à peine une semaine après la visite de Hu Jintao au Quotidien du Peuple en juin 2008. Ces émeutes ont été déclenchées à la suite du viol et du meurtre d'une jeune fille, que la police avait fait passer pour un suicide pour protéger un personnage influent. La population était alors descendue dans la rue pour protester contre les abus répétés des dirigeants locaux, et la situation a dégénéré jusqu'à l'affrontement avec les autorités et le recours à la police armée. En quelques jours, tout le pays a été informé par Internet des événements de Weng'an, obligeant les autorités à sortir de l'attitude de déni qu'elles avaient d'abord adopté. Le secrétaire du Parti de la province du Guizhou s'est alors rendu en personne à Weng'an et a désavoué les autorités locales, s'excusant publiquement du fait qu'elles n'avaient pas prêté une attention suffisante aux préoccupations du public, à la défense de leurs droits et de leur sécurité. Le chef du district, le secrétaire du Parti du district et le chef de la police ont notamment été limogés. Il s'agissait alors de rétablir l'ordre et la 
légitimité du rôle dirigeant du Parti au niveau central, aux frais des dirigeants exerçant à l'échelle du district.

Qian Gang, porteur du China Media Project à l'université de Hong Kong, a également donné en 2009 un exemple de la manière dont la presse officielle relevant du niveau central pouvait se saisir de dénonciations relayées sur Internet pour accabler des dirigeants locaux corrompus. L'agence officielle Xinhua avait en effet relayé des investigations sur un cas d'empoisonnement massif au plomb, d'abord menées par un journal local secondaire, puis publiées en ligne, et qui avaient été passées sous silence dans le Quotidien du Shaanxi. Il notait ainsi que la notion de «supervision par l'opinion publique » pouvait en fait s'exercer dans le sens d'une surveillance et d'une discipline des administrations locales par l'État central, au moyen de la presse et de la manipulation de l'opinion publique (Qian, 2009).

Ces observations rejoignent ainsi les conclusions de Lagerkvist sur la mise en place des sites de e-administration dans la première moitié des années 2000, qui s'est faite notamment sous l'impulsion de fonctionnaires et dirigeants réformateurs, qu'il appelle «technocadres » pour leur enthousiasme vis-à-vis des nouvelles techniques de management public et notamment des nouvelles technologies de l'information. Ces personnels mettent en avant l'importance de moderniser la bureaucratie chinoise, de la rendre plus efficace et plus rentable, mais aussi plus transparente et responsable (accountable), afin de recrédibiliser le rôle de l'État et de garantir sa pérennité. En se basant sur plusieurs études de cas, il souligne cependant à quel point ces initiatives révèlent des tensions entre l'échelon local et l'échelon central, soit que des conflits d'intérêts se fassent jour, soit que les dirigeants locaux cherchent à innover de manière autonome (Lagerkvist, 2005b).

\section{À partir de 2010 : le contrôle « décomplexé » de l'opinion publique}

\section{Prémisses d'un renforcement du contrôle sous l'administration de Hu Jintao}

L'orientation de la gestion de l'opinion publique en ligne a beaucoup changé dans une période plus récente, dans un climat politique sensible, avec l'accession à la présidence de Xi Jinping lors du $18^{\mathrm{e}}$ congrès du Parti communiste chinois en 2012. Xi Jinping a surpris par la tournure très autoritaire et personnalisée qu'il a donnée à la direction des affaires du pays, en engageant notamment des campagnes de répression contre des défenseurs des droits, des avocats et le secteur des ONG en particulier. Il a également engagé une campagne anticorruption d'une durée sans précédent, qui est toujours en cours en 2017, et qui a touché jusqu'au plus haut niveau de l'État avec notamment l'arrestation de Zhou Yongkang, ancien membre du Bureau politique du Parti communiste chinois et ancien ministre de la Sécurité publique (Yuen, 2014). Cette campagne, conjuguée à la mise en place de plusieurs «groupes dirigeants centraux » pilotés personnellement par Xi Jinping sur des sujets clés, montre une volonté importante de centralisation des pouvoirs.

Mais cette phase de renforcement du contrôle de l'information commence en réalité avant même la prise de pouvoir par Xi Jinping. Dès 2010, le Conseil des affaires de l'État a publié un Livre blanc sur l'Internet en Chine (Bureau d'information du Conseil des affaires de l'État, 2010), dans lequel il rappelle le principe général qui fait d'Internet un bienfait octroyé sous condition de docilité politique et justifie ainsi la censure : «Les citoyens de la République populaire de Chine et les citoyens étrangers, 
les personnes légales et autres organisations sur le territoire chinois ont le droit et la liberté d'utiliser Internet; en même temps, ils doivent obéir aux lois et règlements de la Chine et protéger consciencieusement la sécurité d'Internet». Ce Livre blanc marque sans doute un tournant dans le sens où le contrôle de l'information est désormais assumé tout à fait ouvertement et incorporé clairement dans une stratégie générale d'affirmation de la Chine comme une cyberpuissance, qui revendique sa « souveraineté » numérique, à l'échelle nationale et internationale.

En 2011, Hu Jintao a prononcé de nouveaux discours appelant à contrôler Internet plus strictement (Bandurski, 2011). Cela s'est traduit par la création en mai $2011 \mathrm{du}$ Bureau d'État de l'information sur Internet (State Internet Information Office, SIIO), dont les travaux sont directement assurés par le personnel du Bureau de l'information du Conseil des affaires de l'État (State Council Information Office, SCIO), sous la direction de Wang Chen, directeur de ce bureau et directeur adjoint du Département de la propagande du Parti, a constitué un signal significatif de la volonté de piloter Internet de manière ferme et coordonnée. En effet, ce nouveau bureau a pour caractéristique principale de réunir les responsables des principales administrations en charge de la régulation d'Internet, en la personne de deux directeurs adjoints, Xi Guohua, numéro deux du ministère de l'Industrie et des Technologies de l'information (MIIT), et de Zhang Xinfeng, numéro deux du ministère de la Sécurité publique (Xiang Zhang, 2011) (deux autres cadres, Peng Bo et Wang Xiujun, respectivement du SCIO et du MIIT seront nommés directeurs adjoints à temps plein en 2012).

Le fait que les différents aspects de la régulation d'Internet relèvent d'administrations différentes (le MIIT pour les licences des fournisseurs de services, la GAPP pour les informations en ligne, le SARFT pour les contenus audiovisuels, etc.) créait une forme de compétition entre ces différentes instances qui pouvait se traduire par des interférences ou des incohérences. La création de ce bureau révèle donc la volonté de remédier à cet état de fait en centralisant les décisions concernant Internet dans une même administration relevant du plus haut niveau de l'État. Les premières déclarations de Wang Chen, portant sur la nécessité de «mener à bien activement le travail de canalisation de l'opinion publique », montrent qu'il en va bel et bien de la question de la gestion de l'opinion publique, qui est considérée comme centrale (Wang, 2011).

\section{Xi Jinping : la mobilisation politique 2.0}

Xi Jinping va plus loin encore dans cette direction. Le 19 août 2013, il prononce un discours lors d'une Conférence nationale sur le travail de propagande et d'idéologie, lors duquel il martèle l'importance de conduire un travail idéologique dans tous les secteurs, notamment l'éducation, la culture, les organes du Parti, les médias, etc. (China Copyright and Media, 2013). Il annonce ainsi la teneur d'une politique qui donne moins de place à la prise en compte de l'opinion publique, et plus d'importance à son modelage. Cela se traduit en particulier par une exigence très forte d'allégeance des médias officiels. En février 2016, il effectue ainsi, à son tour, une visite des principaux organes de la presse officielle, et il leur enjoint alors de manifester une loyauté totale envers le Parti (Associated Press, 2016).

$\mathrm{Xi}$ met ici bien plus l'accent sur le développement d'une propagande innovante, comme l'a clarifié un article du Quotidien du Peuple relatant la tenue d'une Conférence nationale sur le travail de propagande en ligne, les 5 et 6 janvier 2016 (Bandurski, 2016a), que sur la modernisation de l'État via les technologies de la 
communication, même si naturellement cette dimension reste à l'œuvre. Comme l'illustre une compilation des discours de Xi produite par le Quotidien du Peuple, la notion de guidage de l'opinion publique tient de nouveau une place centrale dans le rapport de Xi Jinping aux médias, et Internet est désormais le vecteur clé de ce guidage (Bandurski, 2016b).

Sur le plan institutionnel, Xi crée un Groupe dirigeant central pour la cybersécurité et l'informatisation en 2014, qu'il pilote lui-même, et renforce l'indépendance du SIIO, renommé en anglais Cyberspace Administration of China (CAC), et au sein duquel est établi le bureau du Groupe dirigeant central. La CAC absorbe également plusieurs institutions qui relevaient auparavant d'autres administrations, comme le CNNIC (Chinese Internet Network Information Center, en charge des noms de domaine et producteur de statistiques), l'Internet Society of China. Elle concentre ainsi des aspects essentiels de la gouvernance d'Internet au sein d'un système coordonné et qui répond directement au plus haut niveau de l'État, même si cette centralisation ne peut résoudre complètement les difficultés liées à la mise en place des politiques par des ministères et agences variés. Les nominations en son sein montrent à quel point la propagande, et donc la gestion de l'opinion publique en ligne, est centrale dans cette réorganisation : dès $2013 \mathrm{Lu}$ Wei prend la direction de ce qui est encore le SIIO, et s'adjoint un troisième directeur adjoint, Ren Xianliang. Les deux hommes ont fait leur carrière au Département de la propagande (Creemers, 2015b, p. 8). Lu Wei, à travers de nombreuses apparitions publiques, y compris à l'international, donne une personnalisation et une visibilité particulièrement fortes à l'action de la CAC. À son départ en 2016, il est également remplacé par un dirigeant issu du Département de la propagande, $\mathrm{Xu}$ Lin.

$\mathrm{Au}$ cours de cette période, la censure d'Internet semble s'être considérablement accrue, de même que la répression des dissidents est devenue particulièrement sévère, surtout dans les milieux de la culture, du journalisme, mais aussi des ONG et des avocats. En 2013, la Cour populaire suprême publie une «interprétation » criminalisant la publication de «rumeurs » sur Weibo, si ces informations sont vues plus de 5000 fois ou relayées plus de 500 fois (Kaiman, 2013). Comme ces éléments ne sont pas contrôlables par les auteurs des messages, cela représente une forte source d'intimidation et d'autocensure. En janvier 2015, l'accès à la messagerie Gmail devient quasiment impossible sans le recours à des systèmes de contournement tels que des VPN. Ce blocage prend une importance symbolique puisqu'il freine les échanges avec des correspondants internationaux. Même Fang Bingxing, l'ingénieur connu pour avoir créé le système de censure chinois, a dû recourir à un VPN en public lors d'une conférence (HAL 90210, 2016). En somme, malgré la sophistication croissante des technologies de filtrage, qui devrait permettre une censure plus « invisible », il semble qu'à certaines occasions on ne prend plus la peine de cacher les traces de la censure. La lenteur des connexions internationales, et les difficultés d'accès à de nombreux services d'utilité quotidienne, sont donc parfois vécues comme un rappel ostentatoire de l'autorité du régime sur la circulation de l'information.

La propagande également se dote d'une stratégie en ligne plus réfléchie, par exemple avec la consigne de privilégier les modes d'expression compassionnels et avec une stratégie de cooptation (ou de pression sur) des leaders d'opinion (A. K. Li, 2015). La technique de l'astroturfing, qui consiste à noyer les conversations en ligne de commentaires favorables au régime, était déjà en place depuis longtemps, mais elle est désormais orientée plus stratégiquement pour «distraire» les internautes des sujets de controverses, et non pour les contredire (King, Pan, Roberts, 2017). Les 
réseaux sociaux sont même mis à contribution pour diffuser des vidéos ou des animations mettant en scène Xi lui-même et son épouse, surnommés «Xi Dada et Pang Mama », travaillant ainsi à la personnalisation du pouvoir sur un mode à la fois humoristique, paternaliste et avec sans doute une touche de second degré, qui n'est pas sans résonance avec l'esprit prompt à la dérision de la jeunesse chinoise connectée.

Ce travail de propagande est désormais assumé et même revendiqué, au point que le Département de la propagande de la Ligue de la jeunesse communiste a pu récemment diffuser une vidéo à la gloire des internautes qui « apportent leur graine » au travail de diffusion d'une "pensée positive», sans en attendre aucune rétribution (ceci par opposition aux personnes rémunérées pour leurs interventions dans les discussions en ligne, désignées par l'appellation péjorative l'Armée des 50 centimes) (Bandurski, 2016c). Internet devient ici un instrument de mobilisation politique des citoyens (dont l'efficacité est néanmoins sujette à caution).

Le régime affiche au demeurant l'ambition de mobiliser les nouvelles technologies à des fins de contrôle très resserré de la population. Les programmes concernant le numérique, tels que «Internet + », qui consiste à encourager la numérisation et le recours aux nouvelles technologies de la communication dans tous les secteurs économiques, et plus encore l'idée de mettre en place un «système de crédit social » (social credit system), en regroupant toutes les sources de données disponibles sur les individus dans une même base de données, laissent penser que le gouvernement chinois a l'ambition de structurer son contrôle de la population au moyen des outils numériques (même si cela représente encore un défi technologique) (Creemers, 2015a). La réflexion semble aujourd'hui plus tournée autour de la mise en place d'un panoptique numérique, dans une perspective de gouvernement paternaliste et autoritaire, qu'autour de l'impératif de rendre l'action publique plus attentive aux demandes de la population et plus transparente.

\section{Conclusion}

En Chine, l'opinion publique fut longtemps considérée comme un élément plutôt passif et qu'il convenait de «guider» par la propagande, mais qui pouvait, à l'occasion, être mobilisée au service du Parti, à des fins de renseignement ou dans le cadre des campagnes politiques. Les médias dits «traditionnels» (presse écrite, télévision, radio), ainsi que le secteur de la culture, et en fait tous les éléments de l'appareil bureaucratique (éducation, unité de travail, etc.), ont joué un rôle essentiel dans l'influence, mais également dans la collecte d'informations sur cette opinion publique. À l'heure de la commercialisation du secteur des médias (Zhao, 2008) et de l'émergence d'Internet, la parole publique devenant plus spontanée, plus interactive, moins facile à appréhender et à manipuler, et dans un "marché des idées (ou un champ de bataille) » de plus en plus pluraliste, le Parti communiste est mis au défi de redéfinir les objectifs et les moyens du contrôle de l'opinion publique (Brady, Wang, 2009, p. 785).

À travers les évolutions de la doctrine officielle concernant la gestion de l'opinion publique en ligne, se dessine le pari, pris par l'administration $\mathrm{Hu}$, que cette opinion publique, canalisée, puisse être mise au service d'un mode de gouvernement technocratique, et instrumentalisée dans la mise au pas d'un appareil politicoadministratif difficile à maîtriser.

Un premier enjeu est celui du renouvellement de la légitimité du parti au pouvoir et donc la perpétuation du régime, sous condition d'une modernisation de ses techniques 
de gouvernance, notamment par une meilleure prise en compte des préoccupations de la population. Un second enjeu est celui de l'entrée de la Chine dans une «discipline » technocratique (au sens de Foucault), à travers l'adoption d'outils de gestion tels que la mesure de l'opinion publique, les dispositifs de e-administration, ou des exigences de transparence (souvent illusoires) de l'action publique (Howell, 2004). Cela s'inscrit dans un mouvement global de diffusion de modes de «gouvernance » et de bonnes pratiques de gestion des affaires publiques qui est critiqué de manière croissante pour la dissimulation et la neutralisation des questions proprement politiques (Hermet, Kazancigil, Prud'homme, 2005). Ainsi, la question soulevée à ce moment-là est non pas la réduction au silence de l'opinion publique en ligne au profit d'un État tout puissant, mais plutôt l'instrumentalisation d'une certaine forme d'expression populaire canalisée, dans la perspective d'une neutralisation de la participation politique qui affecte aussi bien les dirigeants que la population. Cela rejoint les observations de Froissart (2014), ou encore de Teets (2013), qui montrent comment l'État chinois a travaillé à inclure la société civile dans son fonctionnement, dans une forme de «symbiose contingente» (Spires, 2011), et soulignent ainsi l'ambiguïté de ces régimes autoritaires.

Dans une période plus récente, le traitement de l'opinion publique s'inscrit au contraire dans un contexte d'effort de concentration des pouvoirs autour de la personne de Xi Jinping, où la censure est assumée, voire revendiquée par le pouvoir, avec un retour à une conception plus conservatrice du rapport aux médias, qui n'est pas sans rappeler, par certains aspects, la période maoïste.

La perte de libertés sur les plateformes d'information a été palpable depuis 2013, avec un affadissement général des contributions sur la plateforme Weibo tandis que beaucoup d'utilisateurs se tournaient vers la plateforme semi-privative Wechat (Weixin en chinois), qui est tout aussi surveillée et offre moins de visibilité aux publications.

Désormais dotée d'instruments de mesure et de traitement des données extrêmement puissants (et avec toutes les perspectives offertes par le big data), l'administration de Xi Jinping semble privilégier une approche plus panoptique de l'opinion publique en ligne et de l'ensemble des activités qui font l'objet de traces numériques, ce qui prolongerait la capacité du pouvoir central à organiser et discipliner l'appareil d'État, mais sans nécessairement mettre l'accent sur la responsabilité de l'État vis-à-vis des citoyens ou sur la transparence. Ainsi, la combinaison entre ce contrôle accru de l'information et le maintien de la ligne de la modernisation (par exemple avec le projet de numérisation généralisée des activités humaines sous la bannière «Internet $+»)$ (Creemers, 2015a) est particulièrement saisissante. La mise en œuvre de ce programme demandera néanmoins à être observée de près, car il y a de nombreux défis à relever pour que ces ambitions puissent prendre forme, en termes technologiques (comment rendre les données interopérables), comme en termes organisationnels (comment surmonter les résistances et les conflits d'intérêts entre administrations et entre les différents échelons des administrations).

La mise de côté de l'argument central selon lequel l'informatisation de la société doit mettre la bureaucratie au service des citoyens, au profit d'un argumentaire plus orienté sur l'ordre public, représente également une prise de risque pour l'administration Xi, qui touche là à l'un des piliers de la légitimation de l'autorité du Parti communiste au pouvoir depuis les années 1990. La reconnaissance de l'importance de l'opinion publique, notamment dans son expression en ligne, avait généré un optimisme important au sein de la société chinoise, optimisme qui semble fondre tandis que les mesures de contrôle se font plus visibles et assumées. En dépit 
de la force que peut représenter une maîtrise plus complète de l'opinion publique en ligne sur le plan technique, un tel découragement pourrait représenter une faiblesse sur le plan du soutien populaire à la légitimité du régime.

\section{Bibliographie}

Arsène S., 2011a, «De l'autocensure aux mobilisations », Revue française de science politique, 61 (5), p. 893-915.

Arsène S., 2011b, Internet et politique en Chine, Paris, Karthala.

Associated Press, 2016, «Xi Jinping asks for "absolute loyalty" from Chinese state media », The 19 février 2016 , http://www.theguardian.com/world/2016/feb/19/xi-jinping-tours-chinas-top-statemedia-outlets-to-boost-loyalty (accès le 01/06/2016).

Bandurski D., 2006, "Information Office cadre calls cover-up of negative news "naive" », China Media Project, 17 juillet 2006, http://cmp.hku.hk/2007/07/16/518/ (accès le 21/03/2013).

Bandurski D., 2007, «Supervision by public opinion 與论监督》, China Media Project, 8 juillet 2007, http://cmp.hku.hk/2007/07/08/432/ (accès le 21/03/2013).

Bandurski D., 2008a, «China's Guerrilla War for the Web », Far Eastern Economic Review, https://web.archive.org/web/20090601093526/http://www.feer.com:80/essays/2008/a ugust/chinas-guerrilla-war-for-the-web (accès le 05/05/2017).

Bandurski D., 2008b, «Propaganda leaders scurry off to carry out the "spirit" of $\mathrm{Hu}$ Jintao », China Media Project, 25 juin 2008, http://cmp.hku.hk/2008/06/25/1079/ (accès le 14/10/2010).

Bandurski D., 2010a, "How officials can spin the media », China Media Project, 19 juin 2006, http://cmp.hku.hk/2010/06/19/6238/ (accès le 21/03/2013).

Bandurski D., 2010b, « Lose public opinion and we lose it all », China Media Project, 2 novembre 2010, http://cmp.hku.hk/2010/11/02/8448/ (accès le 21/03/2013).

Bandurski D., 2010c, «Open letter from Party elders calls for free speech », China Media Project, 13 octobre 2010, http://cmp.hku.hk/2010/10/13/8035/ (accès le 24/04/2013).

Bandurski D., 2011, «Hu calls for stronger web controls », China Media Project, 21 février 2011, http://cmp.hku.hk/2011/02/21/10152/ (accès le 15/04/2013).

Bandurski D., 2016a, «A "Year of Innovation" for Internet Controls », China Media Project, 7 janvier 2016, http://cmp.hku.hk/2016/01/07/39575/ (accès le 01/06/2016).

Bandurski D., 2016b, «How Xi Jinping Views the News », China Media Project, 3 mars 2016, http://cmp.hku.hk/2016/03/03/39672/ (accès le 01/06/2016).

Bandurski D., 2016c, «Three Cheers for China's Cyber-Volunteers », China Media Project, 13 avril 2016, http://cmp.hku.hk/2016/04/13/39684/ (accès le 01/06/2016).

Bennett W. L., 1998, «The UnCivic Culture: Communication, Identity, and the Rise of Lifestyle Politics », PS: Political Science and Politics, 31 (4), p. 741-761. 
Bloomberg, 2011, «China's Spending on Internal Policing Outstrips Defense Budget », Bloomberg, http://www.bloomberg.com/news/articles/2011-03-06/china-sspending-on-internal-police-force-in-2010-outstrips-defense-budget (accès le 31/05/2016).

Brady A., Wang W., 2009, "China's Strengthened New Order and the Role of Propaganda », Journal of Contemporary China, 18 (62), p. 767-788.

Bureau d'information du Conseil des affaires de l'État, 2010, White paper: the Internet in China, http://china.org.cn/government/whitepaper/node_7093508.htm (accès le 23/06/2010).

Bureau politique du Comité central du Parti communiste chinois, 1992, «Opinions concerning Strengthening and Improving Propaganda and Ideology Work, and Even Better Serving Economic Construction and Reform », China Copyright and Media (trad.), http://chinacopyrightandmedia.wordpress.com/1992/09/03/opinionsconcerning-strengthening-and-improving-propaganda-and-ideology-work-and-evenbetter-serving-economic-construction-and-reform/ (accès le 14/04/2014).

Chan A., 2007, «Guiding Public Opinion Through Social Agenda Setting: China's media policy since the 1990s », Journal of Contemporary China, 16 (53), p. 547-561.

China Copyright and Media, 2013, «Xi Jinping's 19 August speech revealed? (Translation)», China Copyright and Media, 12 novembre 2013, https://chinacopyrightandmedia.wordpress.com/2013/11/12/xi-jinpings-19-augustspeech-revealed-translation/ (accès le 01/06/2016).

CNNIC，2017, 中国互联网络发展状况统计报告 [Rapport statistique sur le développement d'Internet en Chine], Pékin, CNNIC, http://www.cnnic.net.cn/hlwfzyj/hlwxzbg/hlwtjbg/201701/P02017012336467265740 8.pdf (accès le 08/05/2017).

Conseil des affaires de l'État, 1994, «Computer Information System Security Protection Regulations of the People's Republic of China » (trad.), China Copyright and Media, http://chinacopyrightandmedia.wordpress.com/1994/02/18/computerinformation-system-security-protection-regulations-of-the-peoples-republic-of-china/ (accès le 16/04/2014).

Conseil des affaires de l'État, 1996, «Provisional Computer Information Network International Interconnection Management Regulations of the People's Republic of China » (trad.), China Copyright and Media, http://chinacopyrightandmedia.wordpress.com/1997/05/20/provisional-computerinformation-network-international-interconnection-management-regulations-of-thepeoples-republic-of-china/ (accès le 16/04/2014).

Conseil des affaires de l'État, 2000a, « Regulation on Internet Information Service of the People's Republic of China» (trad.), China Copyright and Media, http://chinacopyrightandmedia.wordpress.com/2000/09/25/internet-informationservice-management-rules/ (accès le 16/04/2014).

Conseil des affaires de l'État, 2000b, «Regulation on Telecommunications » (trad.), World Internet Property Organization, http://www.wipo.int/wipolex/en/details.jsp?id=6560 (accès le 23/04/2013).

Creemers R., 2015a, «Internet Plus: Technology at the Centre of Chinese Politics », China Analysis - Les Nouvelles de Chine, numéro special, juillet 2015, p. 3-6, 
http://www.ecfr.eu/page/-/CA_1507_Governing_the_Web.pdf (accès le 06/06/2016).

Creemers R., 2015b, «Le pivot de la cybergouvernance chinoise », Perspectives chinoises, 4, p. 3-14.

Froissart C., 2014, «The Ambiguities between Contention and Political Participation: A Study of Civil Society Development in Authoritarian Regimes », Journal of Civil Society, 10 (3), p. 219-222.

HAL 90210, 2016, «Father of the Great Firewall of China blocked by his own creation », The Guardian, 6 avril 2016, https://www.theguardian.com/technology/2016/apr/06/great-firewall-of-chinablocked-fang-binxing (accès le 06/06/2016).

He B., Thøgersen S., 2010, «Giving the People a Voice? Experiments with consultative authoritarian institutions in China », Journal of Contemporary China, 19 (66) p. 675-692.

Hermet G., Kazancigil A., Prud'homme J.-F., 2005, La gouvernance : un concept et ses applications, Paris, Karthala.

Howell J. (dir.), 2004, Governance in China, Oxford, Rowman \& Littlefield.

Hu J., Zheng Y., 2016, «Les "mécanismes diversifiés de résolution des conflits" en Chine contemporaine », Perspectives chinoises, 2, p. 49-58.

Kaiman J., 2013, «China cracks down on social media with threat of jail for "online rumours" », The $\quad 10$ septembre 2013, http://www.theguardian.com/world/2013/sep/10/china-social-media-jail-rumours (accès le 21/01/2014).

King G., Pan J., Roberts M. E., 2017, How the Chinese Government Fabricates Social Media Posts for Strategic Distraction, not Engaged Argument, Cambridge (MA), Harvard, http://gking.harvard.edu/files/gking/files/50c.pdf?m=1463962091 (accès le 05/05/2017).

Lagerkvist J., 2005a, «The Rise of Online Public Opinion in the People's Republic of China », China: An International Journal, 3 (1), p. 119-130.

Lagerkvist J., 2005b, «The Techno-cadre's Dream: Administrative Reform by Electronic Governance in China Today? », China Information, 19 (2), p. 189-216.

Leib E., He B. (dir.), 2006, The Search for Deliberative Democracy in China, New York, Palgrave Macmillan.

Li A. K., 2015, «Vers une méthode plus proactive : le contrôle de l'opinion publique sur les microblogs chinois sous la nouvelle présidence de Xi jinping », Perspectives chinoises, 4, p. 15-24.

Li C., 2003, 《坚持三贴近解决五个问题 [Résoudre les Cinq questions en encourageant les Trois proximités]», Xinhua News, http://news.xinhuanet.com/world/2003-05/20/content_969994.htm (accès le 05/05/2017).

Li D., 2008, «The Weng'an model: China's fix-it governance », Open Democracy, 30 juillet 2008, http://www.opendemocracy.net/article/the-wengan-model-china-s-fixit-governance (accès le 11/01/2011). 
MacKinnon R., 2011, "China's "Networked Authoritarianism" », Journal of Democracy, 22 (2), p. 32-46.

Monnoyer-Smith L., Wojcik S., 2014, «La participation politique en ligne, vers un renouvellement des problématiques ? », Participations, 8, p. 5-29.

Murphy Z., 2011, «China struggles to censor train crash coverage », $B B C, 28$ juillet 2011, http://www.bbc.co.uk/news/world-asia-pacific-14321787 (accès le 25/04/2013).

Nathan A. J., 2003, « Authoritarian resilience », Journal of Democracy, 14 (1), p. 617.

ONI, 2012, «China », OpenNet Initiative, http://opennet.net/research/profiles/china (accès le 05/05/2017).

People's Daily Online, 2008, «Hu Jintao talks to netizens via People's Daily Online », People's Daily Online, 20 juin 2008, http://english.peopledaily.com.cn/90001/90776/90785/6433952.html (accès le 13/12/2012).

People's Daily Online, 2013, « China to construct public opinion polling platform », , People's Daily Online, $1^{\mathrm{er}}$ mars 2013, http://english.people.com.cn/90882/8149024.html (accès le 21/03/2013).

Qian G., 2009, «Central party media "grab the megaphone" », China Media Project, 21 août 2009, http://cmp.hku.hk/2009/08/21/1709/ (accès le 25/04/2013).

Qiang C. Z-W., 2009, Rural informatization in China, Washington DC, World Bank.

Schlæger J., 2013, E-Government in China: Technology, Power and Local Government Reform, Routledge.

Spires A. J., 2011, «Contingent Symbiosis and Civil Society in an Authoritarian State: Understanding the Survival of China's Grassroots NGOs », American Journal of Sociology, 117 (1), p. 1-45.

Tan Z., Mueller M., Foster W., 1997, « China's New Internet Regulations: Two Steps Forward, One Step Back », Communications of the ACM, 40 (12), p. 11-16.

Thireau I., Hua L., 2005, « De l'épreuve publique à la reconnaissance d'un public : le scandale Sun Zhigang », Politix, 3 (71), p. 137-164.

Thireau I., Hua L., 2010, Les ruses de la démocratie : protester en Chine, Paris, Seuil.

Teets J. C., 2013, «Let Many Civil Societies Bloom: The Rise of Consultative Authoritarianism in China », The China Quarterly, 213, p. 19-38.

Tsai W., 2016, «How "Networked Authoritarianism" was Operationalized in China: methods and procedures of public opinion control », Journal of Contemporary China, 25 (101), p. 731-744.

Wang C., 2011, 积极开展微博客舆论引导工作 [Développer activement le travail d'influence de l'opinion sur Weibo], Pékin, Bureau de l'Information du Conseil des affaires de l'État, People.cn, http://politics.people.com.cn/GB/8198/235803/index.html (accès le 05/05/2017).

Wu X., 2007, Chinese cyber nationalism: evolution, characteristics, and implications, Lanham, Lexington Books. 
Xiao Q., 2008, «The Rise of Online Public Opinion and Its Political Impact », in Shirk S. L. (dir.), Changing Media, Changing China, Oxford, Oxford University Press, p. 202-224.

Xuyang J., 2012, «Beijing police launch Internet restrictions », People's Daily Online, 26 juillet 2012, http://english.people.com.cn/90882/7888508.html (accès le 21/03/2013).

Yang G., 2009, The power of the Internet in China: citizen activism online, New York, Columbia University Press.

Yu J., 2012, «Punishing criticisms outdated in today's China », Global Times via People's Daily Online, 12 octobre 2012, http://english.people.com.cn/90882/7974924.html (accès le 21/03/2013).

Yuen S., 2014, «Discipliner le Parti : la campagne anti-corruption de Xi Jinping et ses limites », Perspectives chinoises, 4, p. 45-52.

Zhang Xiang, 2011, «China sets up office for Internet information management » Xinhuanet, 4 mai 2011, http://news.xinhuanet.com/english2010/china/201105/04/c_13857911.htm (accès le 04/03/2013).

Zhang Xiaoling, 2011, The Transformation of Political Communication in China. From Propaganda to Hegemony, Singapour, World Scientific.

Zhao Y., 2008, Communication in China: political economy, power, and conflict, Lanham Md., Rowman \& Littlefield.

\section{Abstract - Online Public Opinion and the New Order of the Chinese regime}

This article relates the doctrines on online public opinion formulated by the Chinese authorities from the late 1990s until today. Through these doctrines, one can analyze the choice of the $\mathrm{Hu}$ administration to use a duly channeled online public opinion to set up a technocratic government, and to instrumentalize it to discipline the Chinese bureaucracy. More recently, censorship of online public opinion was more openly justified, in line with the movement of concentration of power by President Xi Jinping, and with his more conservative policy in the media sector.

Keywords China, Public Opinion, Internet, Propaganda

Séverine Arsène, docteur en science politique de l'IEP de Paris, est chercheuse au CEFC à Hong Kong. Ses recherches sur les usages et la gouvernance d'Internet éclairent les transformations des rapports de pouvoir en Chine. Elle a mené des recherches au laboratoire Orange Labs à Pékin et enseigné à l'Université Lille 3 et à l'Université Georgetown (Washington DC). Son livre, Internet et politique en Chine, a été publié aux éditions Karthala en 2011.

Mots clés Chine, Opinion publique, Internet, Propagande 